\title{
Data-Driven Sequence of Changes to Anatomical Brain Connectivity in Sporadic Alzheimer's Disease
}

\author{
Neil P. Oxtoby ${ }^{1 *}$, Sara Garbarino ${ }^{1}$, Nicholas C. Firth ${ }^{1,2}$, Jason D. Warren ${ }^{2}$, \\ Jonathan M. Schott ${ }^{2}$, Daniel C. Alexander ${ }^{1}$ and For the Alzheimer's Disease \\ Neuroimaging Initiative
}

'Progression of Neurodegenerative Disease Group (POND), Centre for Medical Image Computing, Department of Computer Science, University College London, London, United Kingdom, ${ }^{2}$ Dementia Research Centre, Department of Neurodegenerative Disease, UCL Institute of Neurology, University College London, London, United Kingdom

\section{OPEN ACCESS}

Edited by:

Yasser Iturria Medina,

Montreal Neurological Institute

and Hospital, Canada

Reviewed by:

Gianfranco Spalletta,

Fondazione Santa Lucia

(IRCCS), Italy

Elis Eleutherio,

Federal University of the

State of Rio de Janeiro, Brazil

${ }^{*}$ Correspondence:

Neil P. Oxtoby

n.oxtoby@ucl.ac.uk

Specialty section:

This article was submitted to Neurodegeneration, a section of the journal

Frontiers in Neurology

Received: 14 August 2017 Accepted: 17 October 2017 Published: 07 November 2017

Citation:

Oxtoby NP, Garbarino S, Firth NC,

Warren JD, Schott JM and Alexander DC (2017) Data-Driven Sequence of Changes to Anatomical

Brain Connectivity in Sporadic Alzheimer's Disease.

Front. Neurol. 8:580. doi: 10.3389/fneur.2017.00580
Model-based investigations of transneuronal spreading mechanisms in neurodegenerative diseases relate the pattern of pathology severity to the brain's connectivity matrix, which reveals information about how pathology propagates through the connectivity network. Such network models typically use networks based on functional or structural connectivity in young and healthy individuals, and only end-stage patterns of pathology, thereby ignoring/excluding the effects of normal aging and disease progression. Here, we examine the sequence of changes in the elderly brain's anatomical connectivity over the course of a neurodegenerative disease. We do this in a data-driven manner that is not dependent upon clinical disease stage, by using event-based disease progression modeling. Using data from the Alzheimer's Disease Neuroimaging Initiative dataset, we sequence the progressive decline of anatomical connectivity, as quantified by graph-theory metrics, in the Alzheimer's disease brain. Ours is the first single model to contribute to understanding all three of the nature, the location, and the sequence of changes to anatomical connectivity in the human brain due to Alzheimer's disease. Our experimental results reveal new insights into Alzheimer's disease: that degeneration of anatomical connectivity in the brain may be a viable, even early, biomarker and should be considered when studying such neurodegenerative diseases.

Keywords: brain connectivity analysis, data-driven, Alzheimer's disease, disease progression modeling, graph theory analysis, computational model

\section{INTRODUCTION}

There is a growing body of literature using advanced computational and statistical modeling to understand progressive disorders. The majority of these has been applied to the most common neurodegenerative disorder of the brain, Alzheimer's disease (AD) - see Ref. (1), for a review of the field. Discriminative models range from simple diagnosis using supervised machine-learning classifiers (2-4) to unsupervised clustering of disease subtypes (5-7). Such discriminative models typically do not directly include a notion of disease progression. By contrast, generative data-driven models (8-11) estimate disease progression signatures that can aid disease understanding from start to end. Such an understanding is vital for the early identification of individuals who are likely to be responsive to a particular therapy, and also for identifying the earliest pathological changes for designing such therapies. The protracted duration of $\mathrm{AD}$, and especially the decades-long presymptomatic 
phase, makes this a challenging disorder to study in the general population. Dominantly inherited variants of AD, e.g., Ref. (12)., can be used to alleviate this problem to some extent, but the open question of whether dominantly inherited $\mathrm{AD}$ is a suitable model for sporadic late-onset AD remains unanswered.

Alzheimer's disease has been widely described as a disconnection syndrome (13-16). This has contributed to the motivation behind the relatively recent emergence of network models of neurodegeneration. The motivation is to understand mechanisms of disease propagation by relating models of brain networks to observed pathology. The networks are abstract representations of "connections" between brain regions and can be constructed from (1) functional correlations during rest or activity (usually estimated using blood-oxygen-level-dependent contrast MRI (17)), (2) gray-matter covariance (estimated from structural MRI), or (3) anatomical connections (estimated using neuronal tractography from diffusion-weighted MRI).

Network spreading models attempt to explain neurodegeneration in terms of the transneuronal spread of prion-like (18) pathogens such as abnormal proteins. Anatomical connectivity networks are a natural choice for such models as they estimate physical connections between brain regions, rather than the correlations estimated in functional and gray-matter structural covariance networks. Previous work in network spreading models of neurodegenerative diseases has considered how well these models can predict end-stage disease by: correlating patterns of healthy intrinsic (functional) connectivity and gray-matter volume (14); using healthy functional connectivity to compare network-based mechanistic hypotheses of $\mathrm{AD}$ progression (19); and using healthy anatomical connectivity to predict atrophy (20), amyloid load (21), or metabolism (22). All of these network spreading models used static connectivity of healthy, young individuals to build networks with which to predict end-stage AD pathology. This ignores the effects of aging and disease progression on the network substrate being used to predict pathology.

In contrast to previous network spreading models, we consider elderly connectivity networks, and we explicitly model the course of disease progression. This enables us to investigate how the brain's anatomical connectivity changes with $\mathrm{AD}$ progression. We do this in a novel manner by analyzing regional network (graph-theoretic $(23,24)$ ) measures of brain connectivity in groups of healthy and diseased individuals, within the context of an event-based model $(8,10)$ of disease progression. The model produces a data-driven, fine-grained signature of the sequence of disease-related changes in anatomical connectivity of the human brain, including uncertainty in the sequence. Our innovations beyond earlier event-based models $(8,10)$ include analyzing biomarkers of brain connectivity and employing a new nonparametric mixture modeling technique (25) for estimating biomarker abnormality that is built upon kernel density estimation $(26,27)$.

\section{MATERIALS AND METHODS}

Our analysis can be summarized as three steps. First, we used probabilistic anatomically constrained tractography to construct individual whole-brain connectomes for imaging data from 168 participants from the Alzheimer's Disease Neuroimaging Initiative (ADNI) study, across four age-matched and educationmatched clinical categories $(4 \times 42$ per category, the maximum available in one of the categories). The image analysis pipeline is visualized in the schematic of Figure 1. Second, from each connectome, we computed various local network measures (graph-theory metrics) representing the topology of anatomical connectivity in regions of each participant's brain. Finally, we estimated the ordered sequence in which these measures become abnormal using an event-based model $(8,10)$.

\subsection{Data}

Data used in the preparation of this article were obtained from the Alzheimer's Disease Neuroimaging Initiative (ADNI) database. ${ }^{1}$ The ADNI was launched in 2003 as a public-private partnership, led by Principal Investigator Michael W. Weiner, M.D. The primary goal of ADNI has been to test whether serial magnetic resonance imaging (MRI), positron emission tomography (PET), other biological markers, and clinical and neuropsychological assessment can be combined to measure the progression of mild cognitive impairment (MCI) and early Alzheimer's disease (AD).

In February 2017, we remotely accessed the Laboratory of NeuroImaging's Image Data Archive at the University of Southern California and searched for suitable participants to include in our anatomical connectome cohort: ADNI participants whose brains were imaged with both structural magnetic resonance imaging (MRI) and diffusion-weighted imaging (DWI) at a single study visit. We sought age-matched groups across the four diagnoses of Cognitively Normal (CN), Early Mild Cognitive Impairment (EMCI), Late Mild Cognitive Impairment (LMCI), and probable $\mathrm{AD}(\mathrm{AD})$. This resulted in 42 participants per group. We

$\overline{{ }^{1} \text { http://adni.loni.usc.edu. }}$

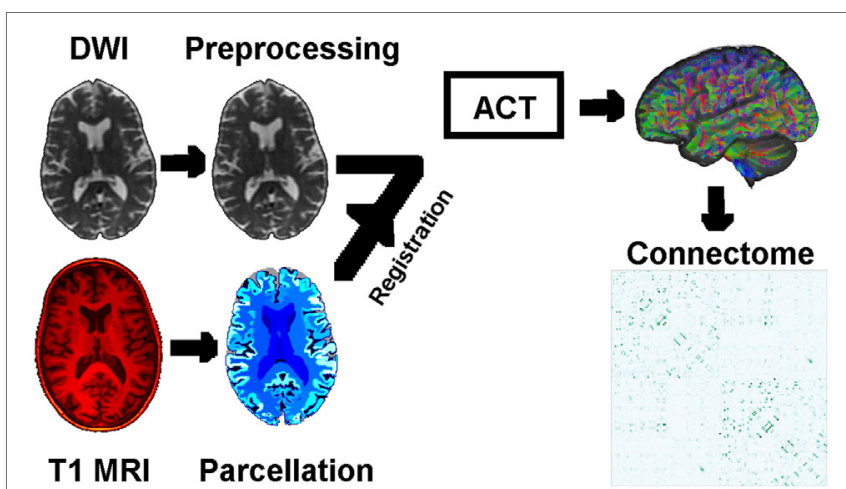

FIGURE 1 | Schematic of the image analysis pipeline, per individual: DWI preprocessing $\rightarrow$ DWI normalization $\rightarrow$ rigid registration of $T 1 \mathrm{MRI}$ to $\mathrm{DWI} \rightarrow \mathrm{T} 1$ parcelation $\rightarrow$ anatomically constrained

tractography $\rightarrow$ connectomes based on density of WM neuronal connections between GM regions of interest. Abbreviations: DWI, diffusion-weighted image; MRI, magnetic resonance image; ACT, anatomically constrained tractography; WM/GM, white/gray matter. 
downloaded the unprocessed structural MRI (3.0T, T1-weighted, non-accelerated IR-SPGR; GE Medical Systems) and DWI (Axial diffusion tensor imaging; GE Medical Systems) for the first available suitable visit of these 168 participants from the ADNI2 phase of ADNI (including: 5 rollovers from the first ADNI phase, ADNI1; and a single rollover from the ADNI Grand Opportunity phase, ADNIGO). Herein, we refer to our anatomical connectome cohort as The168. We also downloaded associated demographics data and metadata in CSV format. Demographics for The168 are summarized in Table 1, and for all ADNI2 (at ADNI2 baseline) in Table 2.

Our disease progression modeling requires both a control and a patient group, which we defined as amyloid-negative $\mathrm{CN}$ participants and amyloid-positive $\mathrm{AD}$ participants, respectively (see bold figures in Table 1). The threshold for amyloid positivity was chosen as an amyloid PET (Florbetapir ${ }^{18} \mathrm{~F}-\mathrm{AV}-45$, hereafter AV45) cut-point from the literature (28): AV45 Standardized Uptake Value Ratio $S U V R \geq 1.10$, which was based on the upper $95 \%$ confidence interval for young healthy subjects. Using this criteria, in The168, we identified 26 controls and 38 patients out of a possible maximum of $42 \mathrm{each}$. For the wider ADNI 2 cohort (including rollovers from ADNI1 and ADNIGO), we found 106 controls and 122 patients-see the bold figures in Table 2.

\subsection{Connectomics}

Structural connectomes were generated using tools provided in the MRtrix3 software package, ${ }^{2}$ customized to work with the Geodesic Information Flows algorithm (29) for segmentation and parcelation. The pipeline included DWI denoising (30),

${ }^{2}$ http://mrtrix.org.

TABLE 1 | Demographics by diagnosis for our anatomical connectome cohort, The168: 168 ADNI2 participants included in this study.

\begin{tabular}{lllll}
\hline & CN & EMCI & LMCl & AD \\
\hline$n$ (Females) & $42(23)$ & $42(17)$ & $42(16)$ & $42(17)$ \\
$n$ APOE+ (APOE-) & $14(27)$ & $20(21)$ & $31(10)$ & $28(13)$ \\
$n$ AV45+ (AV45-) & $15(\mathbf{2 6})$ & $22(17)$ & $29(6)$ & $\mathbf{3 8}(4)$ \\
Age mean (SD) & $73(6)$ & $73(7)$ & $73(7)$ & $73(7)$ \\
Edu mean (SD) & $16(3)$ & $16(3)$ & $16(3)$ & $16(3)$ \\
\hline
\end{tabular}

One-way ANOVA implies that the groups do not differ significantly by age $(p>0.97)$, nor education ( $p>0.45$ ). Amyloid positivity was set at cortical mean AV45

SUVR $\geq 1.10$ (28), prior to adjustment for covariates. Numbers in bold font indicate

controls and patients (see text for details) used in our disease progression modeling.

TABLE 2 | Demographics by diagnosis for all ADNI2 participants at initial visit.

\begin{tabular}{lcccc}
\hline & CN & EMCI & LMCI & AD \\
\hline$n$ (Females) & $336(174)$ & $277(124)$ & $450(183)$ & $276(121)$ \\
$n$ APOE+ (APOE-) & $94(240)$ & $116(157)$ & $236(212)$ & $184(89)$ \\
$n$ AV45+ (AV45-) & $52(\mathbf{1 0 6})$ & $105(100)$ & $98(42)$ & $\mathbf{1 2 2}(15)$ \\
Age mean (SD) & $75(6)$ & $71(7)$ & $74(7)$ & $75(8)$ \\
Edu mean (SD) & $16(3)$ & $16(3)$ & $16(3)$ & $15(3)$ \\
\hline
\end{tabular}

As in Table 1, amyloid positivity was set at cortical mean AV45 SUVR $\geq 1.10$ (28), prior to adjustment for covariates. Numbers in bold font indicate controls and patients (see text for details) used in our disease progression modeling. preprocessing $(31,32)$, and bias field correction (33); inter-modal registration (34); T1 tissue segmentation (29); spherical deconvolution $(35,36)$; probabilistic tractography (37) utilizing anatomically constrained tractography (38) and dynamic seeding (39); spherical deconvolution informed filtering of tractograms (SIFT) (40); T1 parcelation (29); and robust structural connectome construction (41). We used the dwiintens itynorm script in MRtrix3 during DWI preprocessing, but found it necessary to modify the subsequent usage of the population_template script such that DWI masks were not used to create the template (the DWI were pre-masked). Note that we use SIFT to produce biologically plausible tractograms where the streamline density in each voxel matches the fiber orientation distributions estimated from the DWI, instead of simply thresholding the number of fibers connecting two regions.

Our anatomical connectome for each participant is a weighted adjacency matrix that includes only inter-node connections across 130 regions of interest consisting of cortical and subcortical gray-matter regions (including striatal), plus the cerebellum and brain stem. Weights, or connection strengths, are normalized to $[0,1]$, and so represent within-participant anatomical connection density. The image analysis pipeline is visualized in Figure 1. The 130 regions of interest are a subset of those in the labeling protocol used by Geodesic Information Flows $(29,42)$, which is a modified version of the Desikan-Killiany-Tourville protocol (43).

\subsection{Anatomical Connectivity Metrics}

For each participant and region of interest, we calculated 12 brain connectivity metrics from the anatomical connectomes, using the Brain Connectivity Toolbox (24) in MATLAB, after appropriate normalization using the weight_conversion function. The local network metrics fall into the following broad categories:

1. Hubs (basic centrality): degree, strength, degree z-score;

2. Importance (advanced centrality and shortest paths): efficiency, characteristic path length, participation coefficient, betweenness centrality, eigenvector centrality, PageRank centrality (44);

3. Segregation/integration: clustering coefficient, eccentricity.

For each region of interest, the medians of the controls and patients distributions for each network measure were statistically compared using a Mann-Whitney-Wilcoxon rank-sum test. Only measures where $p<0.05 / 12$ (Bonferroni-corrected within region) were retained for further analysis within the event-based model of disease progression-we refer to such biomarkers as having "disease signal."

Note that local efficiency and local clustering coefficient return similar information to each other, as do eigenvector centrality and PageRank centrality, and so one may ask whether we are including redundant information in our models. We argue that we are not, as seen in our results where: (1) local efficiency contained disease signal, whereas local clustering coefficient did not and (2) the centrality measures appear in different positions within the model sequences. 


\subsection{Event-Based Model of Disease Progression}

The event-based model $(\mathrm{EBM})(8,10)$ is a data-driven approach for probabilistically sequencing a cross-sectional set of scalar measurements ("biomarkers") in the order in which they become observably abnormal. In this context, an "event" constitutes a biomarker appearing more abnormal/diseased than normal/healthy. The EBM is able to estimate an average sequence of disease progression events from cross-sectional data because the proportion of abnormal measurements within a cohort will decline in concert with the average ordering. That is, the biomarker that changes earliest (the first disease event) will contain the highest proportion of abnormal measurements (from affected and presymptomatic individuals), and so on. The EBM fuses multiple biomarker measurements across individuals, with the simplest versions assuming a single disease progression sequence for all individuals, as done here. Determining biomarker abnormality in a data-driven manner necessitates mixture modeling within biomarkers, for which we use a new method (25), described below in Section 2.4.1. For convenience, we normalize all biomarkers to "c-scores" (standardized z-scores relative to controls) that increase with abnormality. Otherwise, we used the same fitting procedures as in Ref. (10). Crossvalidation of our EBMs was estimated by refitting the sequence (but not the event measures) to 100 separate bootstrap samples from the data.

We planned to build four EBMs of network connectivity changes, corresponding to the three broad categories of connectivity in Section 2.3, plus all biomarkers together:

- "EBM0"-non-network biomarkers.

- "EBM1"-EBM0 markers, plus biomarkers of anatomical brain network hubs.

- "EBM2"-EBM0 markers, plus biomarkers of anatomical brain network importance: centrality and shortest paths.

- "EBM3"-EBM0 markers, plus biomarkers of segregation/ integration in the anatomical brain network.

- "EBM4"-all biomarkers.

EBM0 acts as a reference point and for investigating consistency with previous EBMs of $\operatorname{AD}(10,45)$ and includes only non-network biomarkers: average cortical level of amyloid (from AV45 PET) and hypometabolism (from fludeoxyglucose (FDG) PET), test score on the Mini-Mental State Examination (MMSE) (46), and structural MRI volumes of the hippocampus, entorhinal area, ventricles, and whole brain. Biomarkers were adjusted for healthy age, education, and gender using regression (residuals method, controls only). Brain volumes were also adjusted for intracranial volume. We did not fit EBM3 because there was no disease signal (see Section 2.3) in regional clustering coefficients nor eccentricity.

\subsubsection{Biomarker Event Measures}

The probability of a biomarker event is fundamental for sequencing the biomarker events into a pathological cascade of disease progression. Since not all patients will have experienced later events, and indeed some controls will have already experienced the earliest events in the cascade, it is necessary to fit a mixture model in order to discover the event probability. Previous EBM analyses $(8,10,45,47-49)$ used mixtures of parametric probability distributions such as Gaussian and uniform. Here, we use a new method (25) that fits a mixture of nonparametric kernel density estimate (KDE) distributions.

\section{RESULTS}

\subsection{Global Connectivity}

Figure 2 shows that global network connectivity in health and disease did not differ significantly in our cohort, The168. That is, we found no significant group-level difference between the 26 controls (AV45-positive CN) and 38 patients (AV45-negative $\mathrm{AD}$ ) across four global brain network metrics: density (connectedness), efficiency (segregation), transitivity (segregation), and assortativity (resilience). The null hypothesis in each Mann-Whitney-Wilcoxon rank-sum test was accepted using a Bonferroni-corrected significance level of $p=0.05 / 4=0.0125$ : density $p=0.0147$; transitivity $p=1$; efficiency $p=0.145$; assortativity $p=0.363$.

\subsection{Event-Based Models}

\subsubsection{Biomarkers}

Figure 3 is a visualization of disease signal in the full set of 38 biomarkers included in the EBMs. The vertical axis is the standardized "c-score" for each biomarker along the horizontal axis: c-score is biomarker value standardized to controls (c.f., $\mathrm{z}$-scores). Group-average lines are shown with individual data points as green crosses for controls and red dots for patients.

\subsubsection{Disease Progression Sequences}

The EBM estimates a data-driven probabilistic sequence of biomarker abnormality, which we visualize as plots of grayscale positional variance (horizontal axis) around the maximumlikelihood ordering (vertical axis). The strongest possible ranking of biomarker abnormality would appear as a black diagonal.

Our first experiment was to build "EBM00"- an EBM including only non-network biomarkers, and using available ADNI2 baseline data (summarized in Table 2). The results are shown in Figure 4, with positional variance estimated from the MCMC fitting procedure (8) shown in the left of the figure, and from bootstrapping shown in the right of the figure (cross-validation). The EBM00 sequence is consistent with current understanding of $\mathrm{AD}$ progression (and previous EBMs (10)): early amyloidosis and hippocampal volume loss, followed by cognitive decline, then hypometabolism and broader neurodegeneration.

In our next experiment, we built "EBM0"-the same biomarkers as EBM00, but using only data from our anatomical connectome cohort, The168 (Table 1). The results are shown in Figure 5, with the positional variance diagram from fitting on the left, and from bootstrapping on the right. Like EBM00, the EBM0 sequence is consistent with current understanding of $\mathrm{AD}$ progression, with only one exception: the apparent late appearance of hypometabolism (FDG). We attribute this to the relatively small number of probable AD patients in The 168 with abnormally 

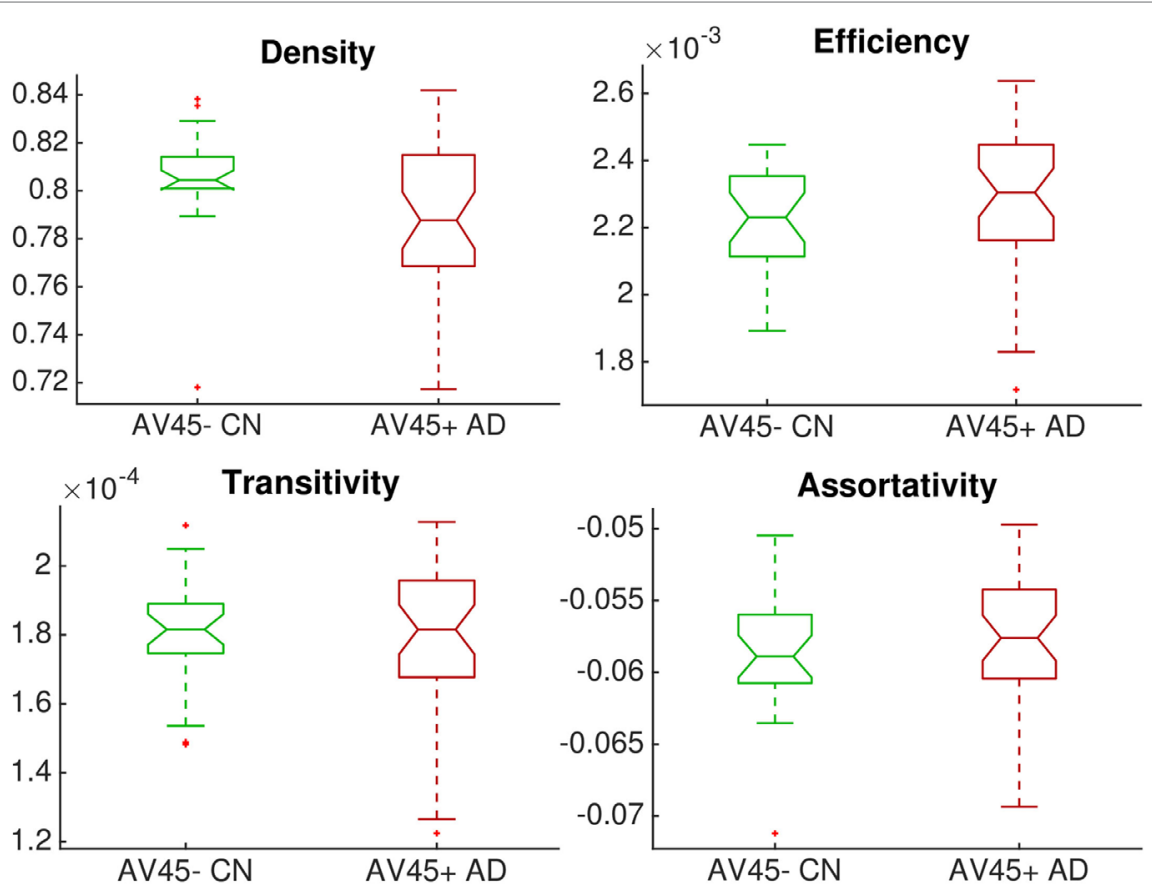

FIGURE 2 | Group comparison of dimensionless global network measures between controls (amyloid-negative CN, green) and patients (amyloid-positive probable $A D$, red). There were no significant differences between groups.

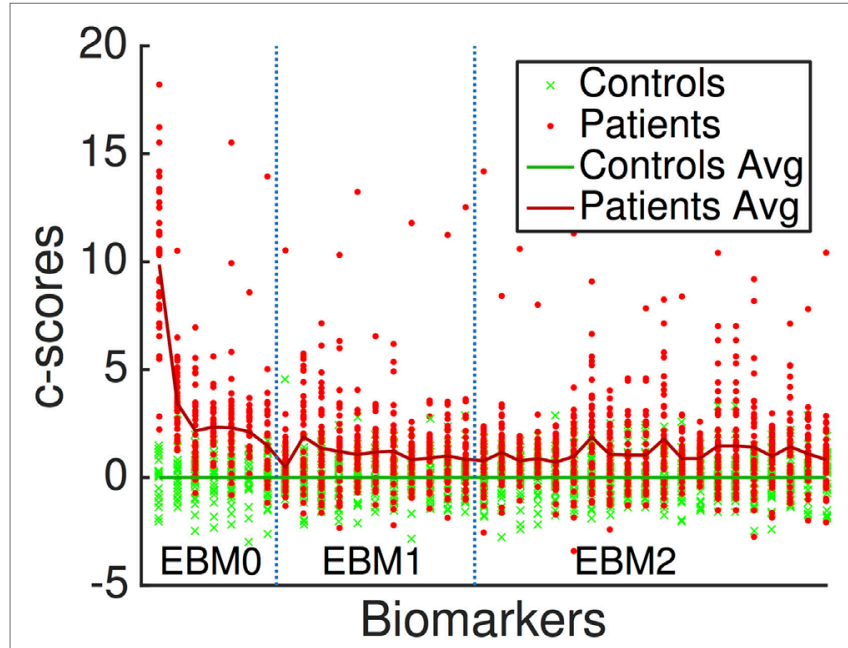

FIGURE 3 | Standardized "c-scores" for all biomarkers included in our analyses. Non-network markers are to the left, denoted EBMO. Network metrics are to the right, delineated by EBM1 (hubs, i.e., basic measures of centrality) and EBM2 (importance, i.e., advanced measures of centrality and shortest paths). EBM3 was not generated since no segregation/integrationbased network measures contained disease signal. EBM4 includes all biomarkers.

high metabolism, compared to the full ADNI2 cohort (Figure S1 in Supplementary Material). We also note that the positional variance is larger in EBM0 than in EBM00, probably due to the lower numbers of individuals.
Next, we report results from experiments on brain connectivity changes in AD. Figure 6 shows "EBM1," which includes network biomarkers that measure hubs in the brain network through node degree or strength. Figure 7 shows "EBM2," which includes biomarkers of network importance as measured by centrality and shortest path metrics. Figure $\mathbf{8}$ is "EBM4" which includes both hubs and centrality. ("EBM3" does not exist because no biomarkers of segregation/integration passed the test for disease signal-see Section 2.3.). The network biomarker event measures are presented in Figure S2 in the Supplementary Material.

From Figure 6 we can infer the ordering the network biomarker event measures (from mixture modeling) are presented in Figure S2 in the Supplementary Material. In which certain anatomical network hubs of the brain deteriorate. We note early involvement of regions in the temporal lobe: the left transverse temporal gyrus (TTG; a.k.a. Heschl's gyrus; auditory cortex) and the left temporal pole (TMP; anterior of the temporal lobe); and a region in the frontal lobe: the right lateral orbital gyrus (LOrG). The model suggests that the later hubs to deteriorate include the middle occipital gyrus (MOG), subcallosal area (SCA), left occipital fusiform gyrus $(\mathrm{OFuG})$, and right middle cingulate gyrus (MCgG). Many, but not all, of these regions are involved in the default mode network (DMN), see, e.g., Ref. (50), and references within.

From Figure 7 we infer the sequential deterioration of regional importance in the anatomical network of the brain, as measured by local centrality and efficiency. The model suggests that centrality declines first in memory-related parts of the $\mathrm{DMN}$ in the temporal and prefrontal lobes: left temporal pole 

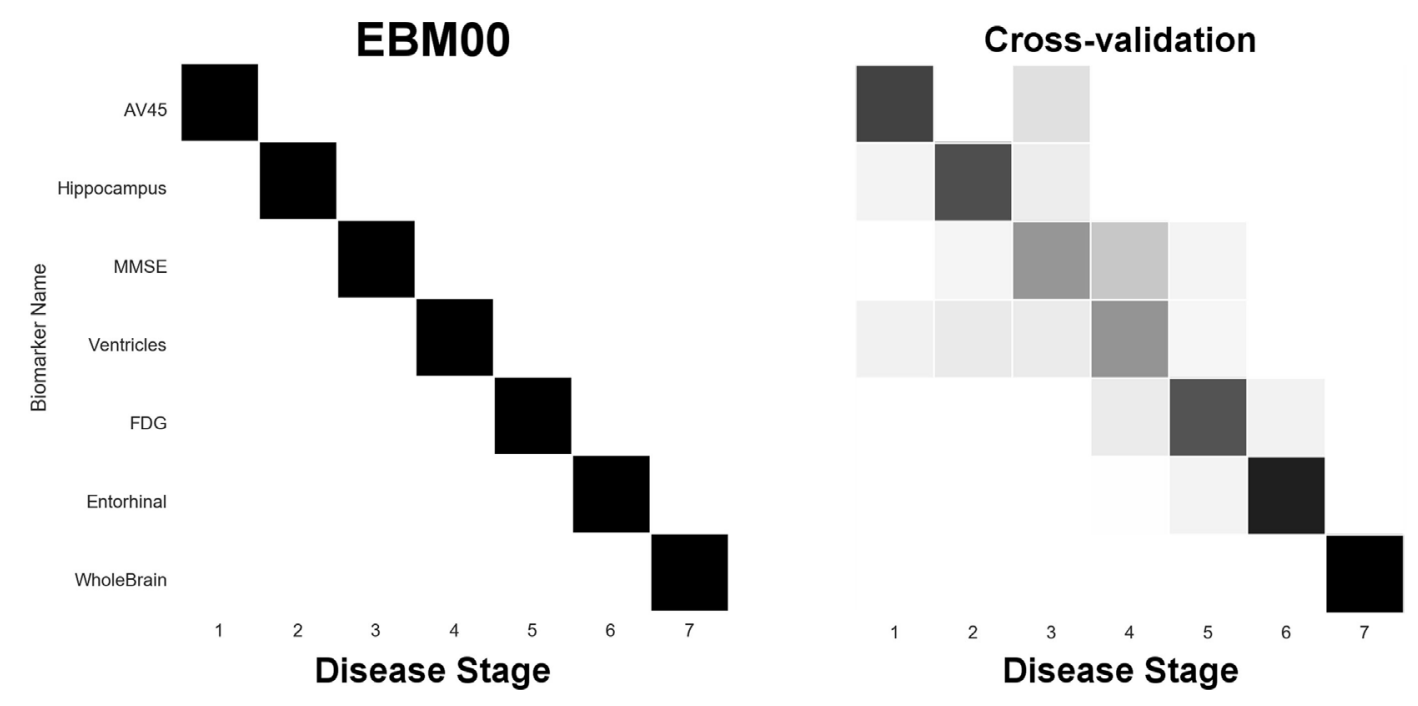

FIGURE 4 | EBM for selected standard (non-network) biomarkers, built on available ADNI2 baseline data. Left panel: left-right positional variance of the maximumlikelihood sequence. Right panel: cross-validation of the sequence from bootstrapping.
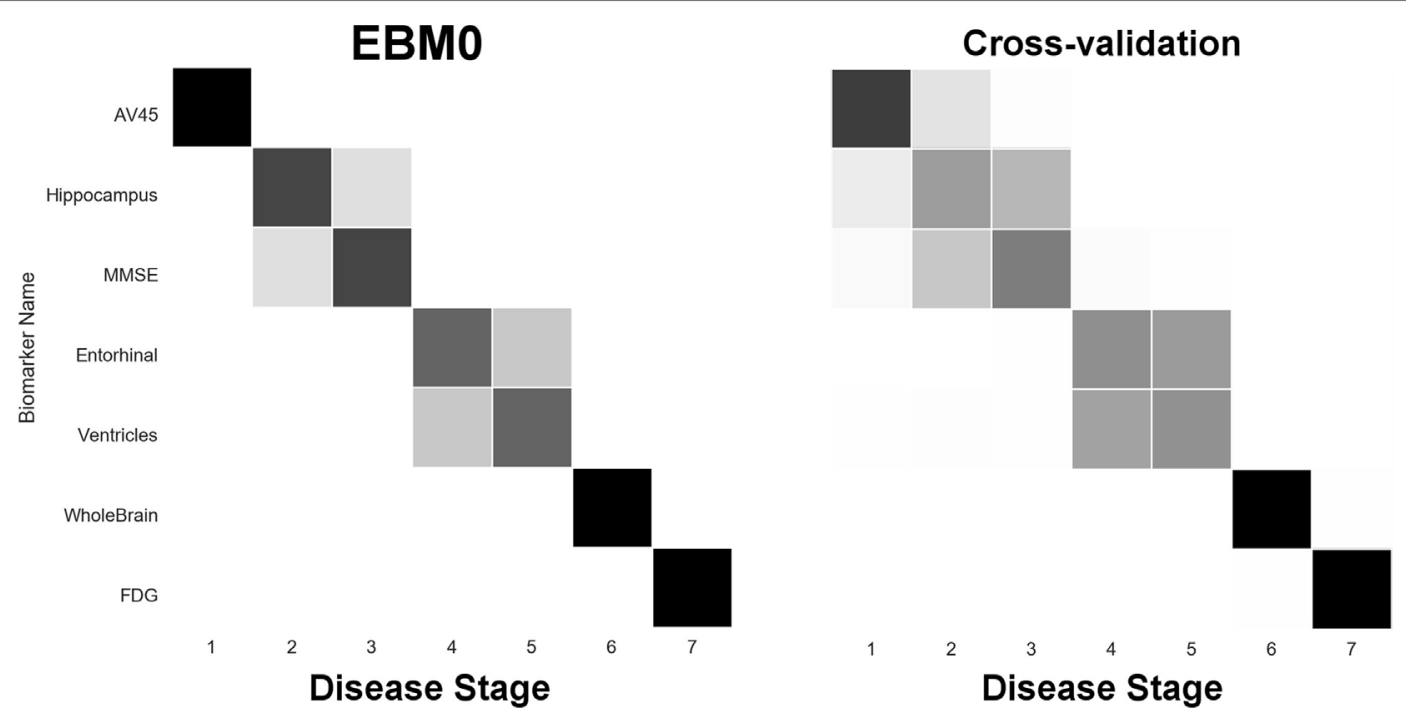

FIGURE 5 | EBM for selected standard (non-network) biomarkers, built on data from our anatomical connectome cohort, The168. Left panel: left-right positional variance of the maximum-likelihood sequence. Right panel: cross-validation of the sequence from bootstrapping.

(TMP), left triangular part of the inferior frontal gyrus (TrIFG), bilateral hippocampus; and last in regions from the occipital (and anterior limbic) lobe(s): right lingual gyrus (LiG; visual cortex), left middle occipital gyrus (MOG), right middle cingulate gyrus (MCgG), and right angular gyrus (AnG).

Finally, Figure 8 ("EBM4") contains all biomarkers of anatomical connectivity included in this study. The ordering of abnormality in hubs and centrality biomarkers is consistent with EBM1 and EBM2, respectively. Figure 8 enables comparison of the relative sequential decline among hubs and regional centrality in the $\mathrm{AD}$ brain's anatomical connectivity network. We observe that the clearest and earliest decline in anatomical connectivity in the $\mathrm{AD}$ brain is both hubs and centrality in the left temporal lobe, all of which are DMN regions known to have memoryrelated storage and functions. Memory dysfunction is the clinical phenotype of typical $\mathrm{AD}$ dementia.

\section{DISCUSSION}

\subsection{Findings in Context}

Brain network hubs are thought to experience increased susceptibility to AD pathology due to higher activity and 

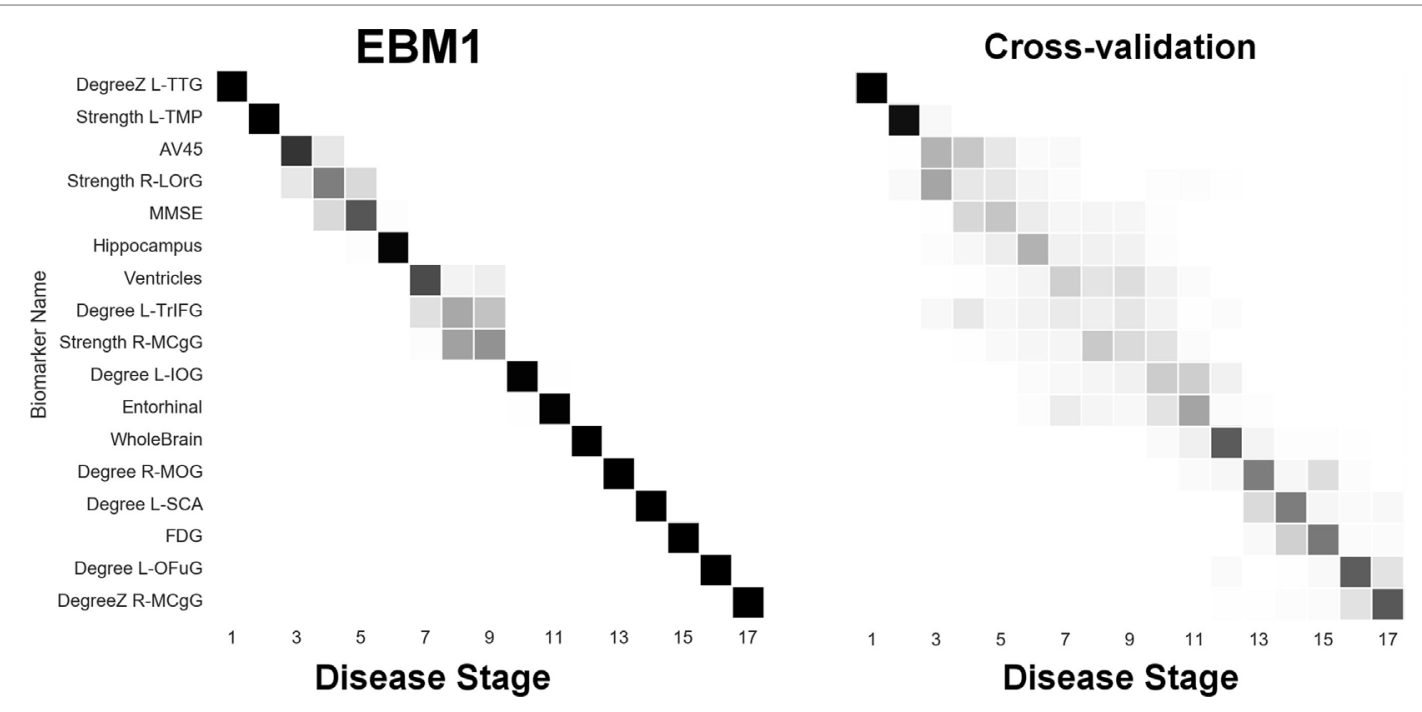

FIGURE 6 | EBM for hubs in the brain's anatomical network, along with selected standard (non-network) biomarkers, built on data from our anatomical connectome cohort, The168. Left panel: left-right positional variance of the maximum-likelihood sequence. Right panel: cross-validation of the sequence from bootstrapping. Abbreviations: DegreeZ, degree z-score; L, left; R, right; TTG, transtemporal gyrus; TMP, temporal pole; LOrG, lateral orbital gyrus; TrIFG, triangular inferior frontal gyrus; MCgG, middle cingulate gyrus; IOG, inferior occipital gyrus; MOG, middle occipital gyrus; SCA, subcallosal area; OFuG, occipital fusiform gyrus.
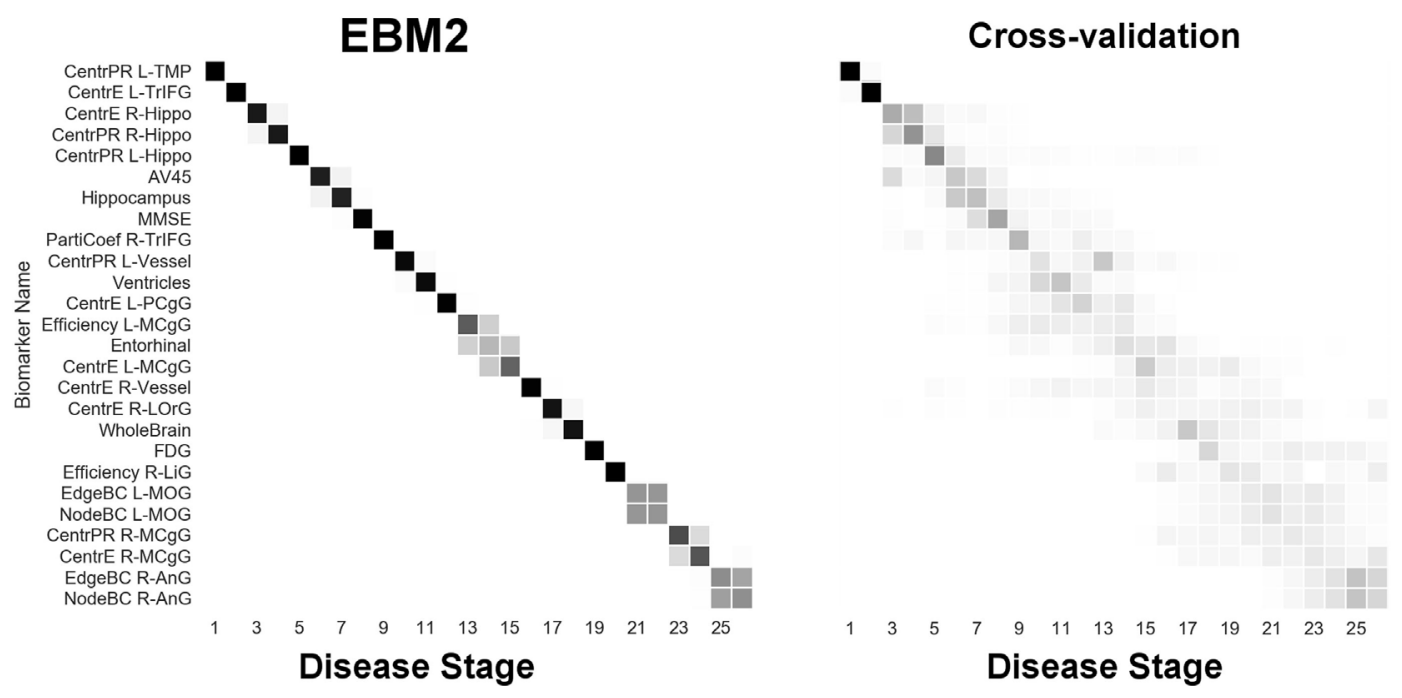

FIGURE 7 | EBM for regional centrality in the brain's anatomical network, along with selected standard (non-network) biomarkers, built on data from our anatomical connectome cohort, The168. Left panel: left-right positional variance of the maximum-likelihood sequence. Right panel: cross-validation of the sequence from bootstrapping. Abbreviations: CentrPR, PageRank centrality; CentrE, eigenvector centrality; PartiCoef, participation coefficient; EdgeBC, edge betweenness centrality; NodeBC, node betweenness centrality; L, left; R, right; TMP, temporal pole; TrlFG, triangular inferior frontal gyrus; Hippo, hippocampus; PCgG, posterior cingulate gyrus; MCgG, middle cingulate gyrus; LOrG, lateral orbital gyrus; LiG, lingual gyrus; MOG, middle occipital gyrus; AnG, angular gyrus.

metabolism, such as regions within the DMN, e.g., Ref. (50), and references within. Our experimental results are consistent with this idea, suggesting that the earliest abnormality in $\mathrm{AD}$ occurs in hubs (Figure 6) and other centrally important regions (Figure 7) in the left temporal lobe, and bilaterally in the hippocampus-see Figure 8. Specific left temporal lobe regions include the transverse temporal gyrus (part of the auditory cortex), the temporal pole (which may be involved in social and emotional cognition, e.g., because it has been shown to be affected in frontotemporal dementia (51)), and the triangular part of the inferior frontal gyrus (which may be involved in semantic memory (52)). 

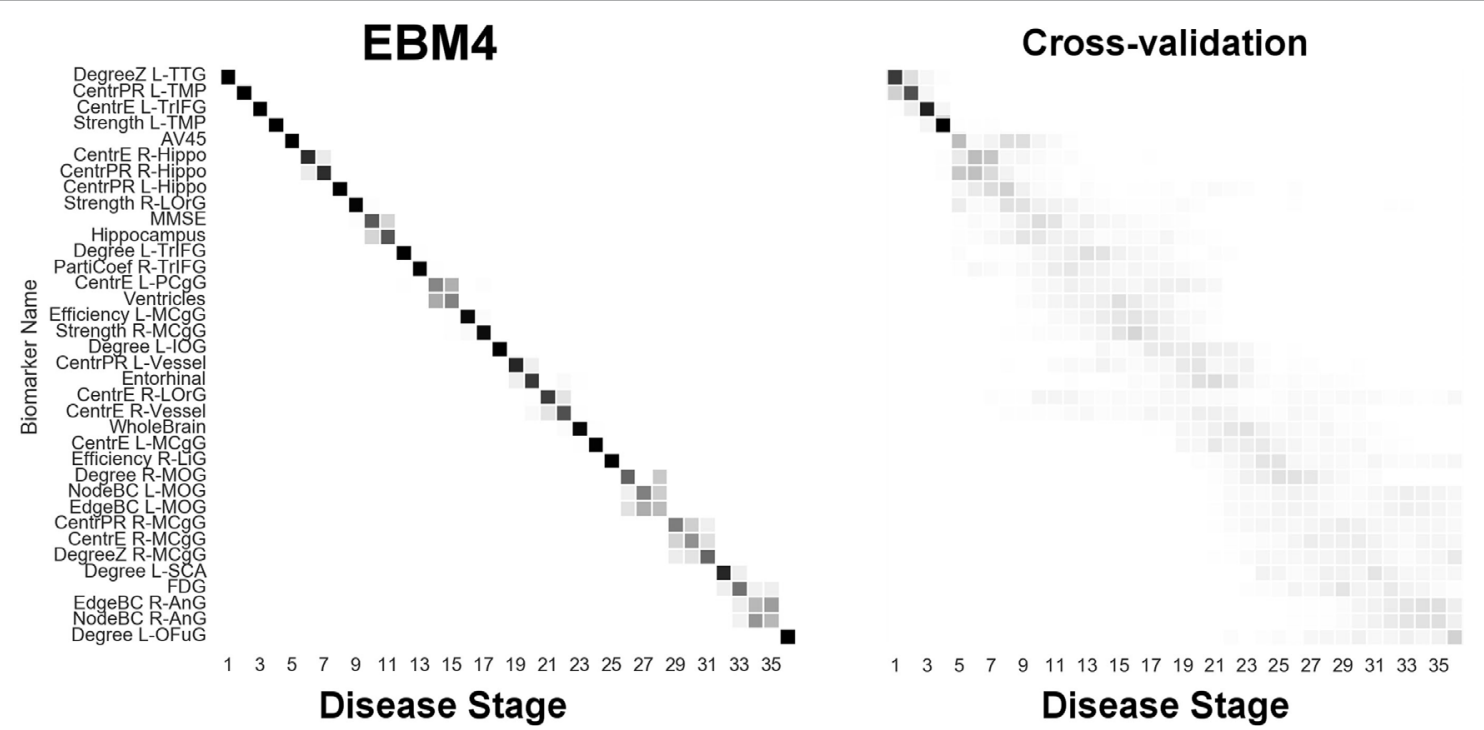

FIGURE 8 | EBM for all biomarkers considered, including hubs and centrality in the brain's anatomical network, along with selected standard (non-network) biomarkers, built on data from our anatomical connectome cohort, The168. Left panel: left-right positional variance of the maximum-likelihood sequence. Right panel: cross-validation of the sequence from bootstrapping. Abbreviations as in Figures $\mathbf{6}$ and $\mathbf{7}$.

Our finding of early connectivity changes involving the auditory cortex is potentially of particular clinical relevance to Alzheimer's disease: hearing impairment has recently emerged as a major risk factor for cognitive decline and a focus of intense epidemiological interest (53), while functional alterations in central auditory processing have been identified in both presymptomatic and established Alzheimer's disease $(54,55)$. Our findings suggest how such alterations may fit within the pathogenic cascade of Alzheimer's evolution.

Our results also suggest that this deterioration of anatomical connectivity in the brain network may be detectable prior to bulk estimates of amyloidosis, such as the mean SUVR across the cortex in AV45 PET. However, prior to claiming that networkbased biomarkers may form sensitive early biomarkers of $\mathrm{AD}$, we would seek confirmation using larger anatomical connectivity cohorts (see future work in Section 4.3).

Our experiments also identified that the anatomical connectivity of some DMN regions is not affected until later in the pathological cascade of AD. These included hubs in the angular gyrus, and hubs and centrality in the medial cingulate gyrus-see Figure 8.

We note that the ordering of brain regions involved in our sequence of anatomical connectivity changes to the Alzheimer's disease brain (Figure 8) does not follow the ordering of regions in the neuropathological sequence identified by Braak and Braak (56). This suggests that changes in anatomical connectivity may not occur in sync with deposition of abnormal protein in brain tissue. Indeed, our results suggest that connectivity changes may appear before bulk deposition of amyloid across the cortex is detectable in vivo.

The brain's anatomical connectivity network is altered by AD. These disease-related connectivity changes may be due to neurodegeneration in gray matter, alterations in deep white matter, or alterations in superficial white matter (57) located near the gray-matter/white-matter boundary. We designed our pipeline to be sensitive to these disease-related alterations by employing anatomically constrained tractography (38) and the SIFT method (40), which should improve the biological accuracy of the tractography-based connectivity estimates.

We have studied the late onset, typical variant of Alzheimer's disease. It will be of interest to apply our technique to explore genetic factors that might influence the progression of Alzheimer's disease; and to young onset Alzheimer's disease where different phenotypic presentations are more commonly seen.

\subsection{Novelty of This Work}

Our approach differs from previous network spreading models in two very important ways. We investigated dynamic pathological disruption of the elderly brain's anatomical connectivity network as a function of $\mathrm{AD}$ progression. Previous network models of $\mathrm{AD}(14,19-22)$ used static connectivity patterns estimated from young and healthy individuals, then employed only end-stage patterns of pathology, such as atrophy, to study network spreading mechanisms. Thus, the previous approaches ignore both the effects of normal aging and the effect of disease progression on the network substrate being used to predict pathology.

Our approach provides utility beyond contributing to our understanding of the AD pathological cascade. These include the ability to estimate a longitudinal disease progression signature that is useful for patient staging (10), and for identification of candidate biomarkers for early diagnosis.

In this respect, we believe that our work may be the first data-driven quantification of the widely held idea that $\mathrm{AD}$ 
progression reflects specific anatomical network disruption, not just neurodegeneration.

\subsection{Future Work}

Future work will include seeking a separate, larger anatomical connectivity cohort upon which to validate our results. While our own cross-validation experiments reported above provide some level of confidence in our conclusions, external validation on a larger cohort would be all the more convincing. Validation on animal models of $\mathrm{AD}$ is a related idea that might be worth pursuing.

Our analyses could be applied to other neurodegenerative diseases. We will actively seek suitable cohorts from other diseases upon which to examine pathological changes in the connectivity of the human brain. This would subsequently extend quite naturally to include application of the models to differential diagnosis, and to the related problem of within-disease subtyping $(7,58)$.

On the highest level, our work involved comparison of graphs (59). There are subtle challenges to comparing graphs and metrics derived from them, even when the number of nodes is constant and the average number of connections is the same (60), as was the case in our study. In the future, we will consider normalizing our connectivity metrics with those derived from random graphs or by looking at distances between graphs (60).

\subsection{Conclusion}

We have sequenced the progressive deterioration of anatomical connectivity in the Alzheimer's disease brain. We believe that this is the first attempt to do so in a data-driven manner that incorporates the effects of both aging and disease progression. Aging is accounted for by using healthy elderly brains as controls. Disease progression is estimated using event-based modeling.

Our experimental results reveal new insights into Alzheimer's disease progression: that degeneration of anatomical connectivity in the brain may be a viable, even early, biomarker and should be considered when studying such neurodegenerative diseases.

\section{AUTHOR NOTE}

Data used in preparation of this article were obtained from the Alzheimer's Disease Neuroimaging Initiative (ADNI) database (http://adni.loni.usc.edu). As such, the investigators within the ADNI contributed to the design and implementation of ADNI and/or provided data but did not participate in analysis or writing of this report. A complete listing of ADNI investigators can be found at: http://adni.loni.usc.edu/wp-content/uploads/ how_to_apply/ADNI_Acknowledgement_List.pdf.

\section{AUTHOR CONTRIBUTIONS}

NO, SG, and DA conceived the study and designed the experiments, in consultation with JS. NO performed the image analysis that produced the connectomes and regional volumes and wrote the manuscript. NO and SG collated the data and performed the event-based modeling analyses with input from NF (in particular the mixture modeling for event measures), JS, and DA. All authors contributed to interpretation of the results and the final version of the manuscript.

\section{ACKNOWLEDGMENTS}

The authors gratefully acknowledge the High Performance Computing team in UCL's Department of Computer Science for providing and maintaining invaluable computational resources.

\section{FUNDING}

NO, SG, JS, and DA are supported by a project that has received funding from the European Union's Horizon 2020 research and innovation programme under grant agreement number 666992. NO acknowledges financial support from The Michael J. Fox Foundation for Parkinson's Research, the Alzheimer's Association, Alzheimer's Research UK, and the Weston Brain Institute through "NetMON: Network Models Of Neurodegeneration” (BAND-15-368107, 11042). JW received grant support from the Alzheimer's Society and the NIHR UCLH Biomedical Research Centre. Data collection and sharing for this project was funded by the Alzheimer's Disease Neuroimaging Initiative (ADNI) (National Institutes of Health Grant U01 AG024904) and DOD ADNI (Department of Defense award number W81XWH-12-2-0012). ADNI is funded by the National Institute on Aging, the National Institute of Biomedical Imaging and Bioengineering, and through generous contributions from the following: AbbVie, Alzheimer's Association; Alzheimer's Drug Discovery Foundation; Araclon Biotech; BioClinica, Inc.; Biogen; Bristol-Myers Squibb Company; CereSpir, Inc.; Cogstate; Eisai, Inc.; Elan Pharmaceuticals, Inc.; Eli Lilly and Company; EuroImmun; F. Hoffmann-La Roche, Ltd. and its affiliated company Genentech, Inc.; Fujirebio; GE Healthcare; IXICO Ltd.; Janssen Alzheimer Immunotherapy Research \& Development, LLC.; Johnson \& Johnson Pharmaceutical Research \& Development LLC.; Lumosity; Lundbeck; Merck \& Co., Inc.; Meso Scale Diagnostics, LLC.; NeuroRx Research; Neurotrack Technologies; Novartis Pharmaceuticals Corporation; Pfizer, Inc.; Piramal Imaging; Servier; Takeda Pharmaceutical Company; and Transition Therapeutics. The Canadian Institutes of Health Research is providing funds to support ADNI clinical sites in Canada. Private sector contributions are facilitated by the Foundation for the National Institutes of Health (http://www.fnih.org). The grantee organization is the Northern California Institute for Research and Education, and the study is coordinated by the Alzheimer's Therapeutic Research Institute at the University of Southern California. ADNI data are disseminated by the Laboratory for Neuro Imaging at the University of Southern California.

\section{SUPPLEMENTARY MATERIAL}

The Supplementary Material for this article can be found online at http://www.frontiersin.org/article/10.3389/fneur.2017.00580/ full\#supplementary-material. 


\section{REFERENCES}

1. Oxtoby NP, Alexander DC, EuroPOND Consortium. Imaging plus X: multimodal models of neurodegenerative disease. Curr Opin Neurol (2017) 30(4): 371-9. doi:10.1097/WCO.0000000000000460

2. Klöppel S, Stonnington CM, Chu C, Draganski B, Scahill RI, Rohrer JD, et al. Automatic classification of MR scans in Alzheimer's disease. Brain (2008) 131(3):681-9. doi:10.1093/brain/awm319

3. Mattila J, Koikkalainen J, Virkki A, Simonsen A, van Gils M, Waldemar G, et al. A disease state fingerprint for evaluation of Alzheimer's disease. J Alzheimers Dis (2011) 27(1):163-76. doi:10.3233/JAD-2011-110365

4. Young J, Modat M, Cardoso MJ, Mendelson A, Cash D, Ourselin S, et al. Accurate multimodal probabilistic prediction of conversion to Alzheimer's disease in patients with mild cognitive impairment. Neuroimage Clin (2013) 2:735-45. doi:10.1016/j.nicl.2013.05.004

5. Whitwell JL, Przybelski SA, Weigand SD, Ivnik RJ, Vemuri P, Gunter JL, et al. Distinct anatomical subtypes of the behavioural variant of frontotemporal dementia: a cluster analysis study. Brain (2009) 132(11):2932-46. doi:10.1093/brain/awp232

6. Nettiksimmons J, DeCarli C, Landau S, Beckett L, Alzheimer's Disease Neuroimaging Initiative. Biological heterogeneity in ADNI amnestic mild cognitive impairment. Alzheimers Dement (2014) 10(5):511.e-21.e. doi:10.1016/ j.jalz.2013.09.003

7. Zhang X, Mormino EC, Sun N, Sperling RA, Sabuncu MR, Yeo BTT, et al. Bayesian model reveals latent atrophy factors with dissociable cognitive trajectories in Alzheimer's disease. Proc Natl Acad Sci U S A (2016) 113(42):E6535-44. doi:10.1073/pnas.1611073113

8. Fonteijn HM, Modat M, Clarkson MJ, Barnes J, Lehmann M, Hobbs NZ, et al. An event-based model for disease progression and its application in familial Alzheimer's disease and Huntington's disease. Neuroimage (2012) 60(3):1880-9. doi:10.1016/j.neuroimage.2012.01.062

9. Jedynak BM, Lang A, Liu B, Katz E, Zhang Y, Wyman BT, et al. A computational neurodegenerative disease progression score: method and results with the Alzheimer's disease neuroimaging initiative cohort. Neuroimage (2012) 63(3):1478-86. doi:10.1016/j.neuroimage.2012.07.059

10. Young AL, Oxtoby NP, Daga P, Cash DM, Fox NC, Ourselin S, et al. A datadriven model of biomarker changes in sporadic Alzheimer's disease. Brain (2014) 137(9):2564-77. doi:10.1093/brain/awu176

11. Donohue MC, Jacqmin-Gadda H, LeGoff M, Thomas RG, Raman R, Gamst AC, et al. Estimating long-term multivariate progression from short-term data. Alzheimers Dement (2014) 10(5):S400-10. doi:10.1016/j. jalz.2013.10.003

12. Bateman RJ, Aisen PS, De Strooper B, Fox NC, Lemere CA, Ringman JM, et al. Autosomal-dominant Alzheimer's disease: a review and proposal for the prevention of Alzheimer's disease. Alzheimers Res Ther (2011) 3(1):1. doi:10.1186/alzrt59

13. Delbeuck X, Van der Linden M, Collette F. Alzheimer's disease as a disconnection syndrome? Neuropsychol Rev (2003) 13(2):79-92. doi:10.1023/ A: 1023832305702

14. Seeley WW, Crawford RK, Zhou J, Miller BL, Greicius MD. Neurodegenerative diseases target large-scale human brain networks. Neuron (2009) 62(1): 42-52. doi:10.1016/j.neuron.2009.03.024

15. Warren JD, Rohrer JD, Schott JM, Fox NC, Hardy J, Rossor MN. Molecular nexopathies: a new paradigm of neurodegenerative disease. Trends Neurosci (2013) 36(10):561-9. doi:10.1016/j.tins.2013.06.007

16. Crossley NA, Mechelli A, Scott J, Carletti F, Fox PT, McGuire P, et al. The hubs of the human connectome are generally implicated in the anatomy of brain disorders. Brain (2014) 137(8):2382-95. doi:10.1093/brain/awu132

17. Ogawa S, Lee TM, Kay AR, Tank DW. Brain magnetic resonance imaging with contrast dependent on blood oxygenation. Proc Natl Acad Sci U S A (1990) 87(24):9868-72. doi:10.1073/pnas.87.24.9868

18. Prusiner SB. A unifying role for prions in neurodegenerative diseases. Science (2012) 336(6088):1511-3. doi:10.1126/science.1222951

19. Zhou J, Gennatas ED, Kramer JH, Miller BL, Seeley WW. Predicting regional neurodegeneration from the healthy brain functional connectome. Neuron (2012) 73(6):1216-27. doi:10.1016/j.neuron.2012.03.004

20. Raj A, Kuceyeski A, Weiner M. A network diffusion model of disease progression in dementia. Neuron (2012) 73(6):1204-15. doi:10.1016/j.neuron. 2011.12.040
21. Iturria-Medina Y, Sotero RC, Toussaint PJ, Evans AC, The Alzheimer's Disease Neuroimaging Initiative. Epidemic spreading model to characterize misfolded proteins propagation in aging and associated neurodegenerative disorders. PLoS Comput Biol (2014) 10(11):e1003956. doi:10.1371/journal. pcbi. 1003956

22. Raj A, LoCastro E, Kuceyeski A, Tosun D, Relkin N, Weiner M, et al. Network diffusion model of progression predicts longitudinal patterns of atrophy and metabolism in Alzheimer's disease. Cell Rep (2015) 10(3):359-69. doi:10.1016/j.celrep.2014.12.034

23. Bullmore E, Sporns O. Complex brain networks: graph theoretical analysis of structural and functional systems. Nat Rev Neurosci (2009) 10(3):186-98. doi: $10.1038 / \mathrm{nrn} 2575$

24. Rubinov M, Sporns O. Complex network measures of brain connectivity: uses and interpretations. Neuroimage (2010) 52(3):1059-69. doi:10.1016/j. neuroimage.2009.10.003

25. Firth NC. A data-driven model for estimating decline of cognition, an application to posterior cortical atrophy and typical Alzheimer's disease. (2017).

26. Parzen E. On estimation of a probability density function and mode. Ann Math Statist (1962) 33(3):1065-76. doi:10.1214/aoms/1177704472

27. Rosenblatt M. Remarks on some nonparametric estimates of a density function. Ann Math Statist (1956) 27(3):832-7. doi:10.1214/aoms/1177728190

28. Johnson KA, Sperling RA, Gidicsin CM, Carmasin JS, Maye JE, Coleman RE, et al. Florbetapir (F18-AV-45) PET to assess amyloid burden in Alzheimer's disease dementia, mild cognitive impairment, and normal aging. Alzheimers Dement (2013) 9(5):S72-83. doi:10.1016/j.jalz.2012.10.007

29. Cardoso MJ, Modat M, Wolz R, Melbourne A, Cash D, Rueckert D, et al. Geodesic information flows: spatially-variant graphs and their application to segmentation and fusion. IEEE Trans Med Imaging (2015) 34(9):1976-88. doi:10.1109/TMI.2015.2418298

30. Veraart J, Fieremans E, Novikov DS. Diffusion MRI noise mapping using random matrix theory. Magn Reson Med Sci (2016) 76(5):1582-93. doi:10.1002/ mrm.26059

31. Andersson JL, Skare S, Ashburner J. How to correct susceptibility distortions in spin-echo echo-planar images: application to diffusion tensor imaging. Neuroimage (2003) 20(2):870-88. doi:10.1016/S1053-8119(03)00336-7

32. Andersson JL, Sotiropoulos SN. An integrated approach to correction for off-resonance effects and subject movement in diffusion MR imaging. Neuroimage (2016) 125:1063-78. doi:10.1016/j.neuroimage.2015. 10.019

33. Tustison NJ, Avants BB, Cook PA, Zheng Y, Egan A, Yushkevich PA, et al. N4itk: improved n3 bias correction. IEEE Trans Med Imaging (2010) 29(6):1310-20. doi:10.1109/TMI.2010.2046908

34. Modat M, Cash DM, Daga P, Winston GP, Duncan JS, Ourselin S. Global image registration using a symmetric block-matching approach. J Med Imaging (2014) 1(2):024003. doi:10.1117/1.JMI.1.2.024003

35. Tournier J-D, Calamante F, Gadian DG, Connelly A. Direct estimation of the fiber orientation density function from diffusion-weighted MRI data using spherical deconvolution. Neuroimage (2004) 23(3):1176-85. doi:10.1016/j. neuroimage.2004.07.037

36. Jeurissen B, Tournier J-D, Dhollander T, Connelly A, Sijbers J. Multi-tissue constrained spherical deconvolution for improved analysis of multi-shell diffusion MRI data. Neuroimage (2014) 103:411-26. doi:10.1016/j.neuroimage. 2014.07.061

37. Tournier J-D, Calamante F, Connelly A. Improved probabilistic streamlines tractography by 2 nd order integration over fibre orientation distributions. Proceedings of the International Society for Magnetic Resonance in Medicine. (Vol. 18), (2010). 1670 p.

38. Smith RE, Tournier J-D, Calamante F, Connelly A. Anatomically-constrained tractography: improved diffusion $\{\mathrm{MRI}\}$ streamlines tractography through effective use of anatomical information. Neuroimage (2012) 62(3):1924-38. doi:10.1016/j.neuroimage.2012.06.005

39. Smith RE, Tournier J-D, Calamante F, Connelly A. SIFT2: enabling dense quantitative assessment of brain white matter connectivity using streamlines tractography. Neuroimage (2015) 119:338-51. doi:10.1016/j.neuroimage. 2015.06.092

40. Smith RE, Tournier J-D, Calamante F, Connelly A. SIFT: spherical-deconvolution informed filtering of tractograms. Neuroimage (2013) 67:298-312. doi:10.1016/j.neuroimage.2012.11.049 
41. Yeh C-H, Smith RE, Liang X, Calamante F, Connelly A. Correction for diffusion $\{\mathrm{MRI}$ fibre tracking biases: the consequences for structural connectomic metrics. Neuroimage (2016) 142:150-62. doi:10.1016/j.neuroimage.2016.05.047

42. Prados F, Cardoso MJ, Burgos N, Wheeler-Kingshott CA, Ourselin S. NiftyWeb: web based platform for image processing on the cloud. International Society for Magnetic Resonance in Medicine (ISMRM) 24th Scientific Meeting and Exhibition. Singapore (2016).

43. Klein A, Tourville J. 101 labeled brain images and a consistent human cortical labeling protocol. Front Neurosci (2012) 6:171. doi:10.3389/fnins.2012.00171

44. Page L, Brin S, Motwani R, Winograd T. The Pagerank Citation Ranking: Bringing Order to the Web. Technical Report 1999-66. Stanford InfoLab (1999). Previous number $=$ SIDL-WP-1999-0120.

45. Oxtoby NP, Young AL, Lorenzi M, Cash DM, Weston PSJ, Ourselin S, et al. Model-based comparison of autosomal-dominant and late-onset Alzheimer's disease progression in the DIAN and ADNI studies. Alzheimers Dement (2016) 12(7):668-9. doi:10.1016/j.jalz.2016.06.1514

46. Folstein MF, Folstein SE, McHugh PR. Mini-mental state. J Psychiatr Res (1975) 12(3):189-98. doi:10.1016/0022-3956(75)90026-6

47. Chen G, Shu H, Chen G, Ward BD, Antuono PG, Zhang Z, et al. Staging Alzheimer's disease risk by sequencing brain function and structure, cerebrospinal fluid, and cognition biomarkers. J Alzheimers Dis (2016) 54(3):983-93. doi:10.3233/JAD-160537

48. Venkatraghavan V, Bron EE, Niessen WJ, Klein S. A discriminative event based model for Alzheimer's disease progression modeling. In: Niethammer $M$, Styner M, Aylward S, Zhu H, Oguz I, Yap P-T, Shen D, editors. Information Processing in Medical Imaging: 25th International Conference; IPMI 2017 June 25-30, 2017, Proceedings. Boone, NC, USA: Springer International Publishing (2017). p. 121-33.

49. Oxtoby NP, Young AL, Cash DM, Benzinger TL, Fagan AM, Morris JC, et al. Data-driven models of dominantly-inherited Alzheimer's disease progression. (2017).

50. Buckner RL, Andrews-Hanna JR, Schacter DL. The brain's default network. Ann N Y Acad Sci (2008) 1124(1):1-38. doi:10.1196/annals.1440.011

51. Olson IR, Plotzker A, Ezzyat Y. The enigmatic temporal pole: a review of findings on social and emotional processing. Brain (2007) 130(7):1718-31. doi:10.1093/brain/awm052

52. Binder JR, Desai RH. The neurobiology of semantic memory. Trends Cogn Sci (2011) 15(11):527-36. doi:10.1016/j.tics.2011.10.001
53. Livingston G, Sommerlad A, Orgeta V, Costafreda SG, Huntley J, Ames D, et al. Dementia prevention, intervention, and care. Lancet (2017). doi:10.1016/ S0140-6736(17)31363-6

54. Gates GA, Beiser A, Rees TS, D’Agostino RB, Wolf PA. Central auditory dysfunction may precede the onset of clinical dementia in people with probable Alzheimer's disease. J Am Geriatr Soc (2002) 50(3):482-8. doi:10.1046/ j.1532-5415.2002.50114.x

55. Golden HL, Nicholas JM, Yong KXX, Downey LE, Schott JM, Mummery CJ, et al. Auditory spatial processing in Alzheimer's disease. Brain (2015) 138(1):189-202. doi:10.1093/brain/awu337

56. Braak H, Braak E. Neuropathological stageing of Alzheimer-related changes. Acta Neuropathol (1991) 82:239-59. doi:10.1007/BF00308809

57. Phillips OR, Joshi SH, Piras F, Orfei MD, Iorio M, Narr KL, et al. The superficial white matter in Alzheimer's disease. Hum Brain Mapp (2016) 37(4):1321-34. doi:10.1002/hbm.23105

58. Young AL, Oxtoby NP, Huang J, Marinescu RV, Daga P, Cash DM, et al. Multiple orderings of events in disease progression. In: Ourselin S, Alexander DC, Westin C-F, Cardoso MJ, editors. Information Processing in Medical Imaging, Lecture Notes in Computer Science. (Vol. 9123), Springer (2015). p. 711-22. doi:10.1007/978-3-319-19992-4_56

59. He Y, Evans A. Graph theoretical modeling of brain connectivity. Curr Opin Neurol (2010) 23:341-50. doi:10.1097/WCO.0b013e32833aa567

60. van Wijk BCM, Stam CJ, Daffertshofer A. Comparing brain networks of different size and connectivity density using graph theory. PLoS One (2010) 5(10):e13701. doi:10.1371/journal.pone.0013701

Conflict of Interest Statement: The authors declare that the research was conducted in the absence of any commercial or financial relationships that could be construed as a potential conflict of interest.

Copyright (c) 2017 Oxtoby, Garbarino, Firth, Warren, Schott and Alexander. This is an open-access article distributed under the terms of the Creative Commons Attribution License (CC BY). The use, distribution or reproduction in other forums is permitted, provided the original author(s) or licensor are credited and that the original publication in this journal is cited, in accordance with accepted academic practice. No use, distribution or reproduction is permitted which does not comply with these terms. 